\title{
Stability Analysis of 2D Discrete Linear System Described by the Fornasini-Marchesini Second Model with Actuator Saturation
}

\author{
Richa Negi, ${ }^{1}$ Haranath Kar, ${ }^{2}$ and Shubhi Purwar ${ }^{1}$ \\ ${ }^{1}$ Department of Electrical Engineering, Motilal Nehru National Institute of Technology, Allahabad 211004, India \\ ${ }^{2}$ Department of Electronics and Communication Engineering, Motilal Nehru National Institute of Technology, \\ Allahabad 211004, India \\ Correspondence should be addressed to Richa Negi, richa_negi123@yahoo.co.in
}

Received 7 November 2011; Accepted 3 December 2011

Academic Editors: J. Buick, P. B. Vasconcelos, and Q.-W. Wang

Copyright () 2012 Richa Negi et al. This is an open access article distributed under the Creative Commons Attribution License, which permits unrestricted use, distribution, and reproduction in any medium, provided the original work is properly cited.

This paper proposes a novel antiwindup controller for 2D discrete linear systems with saturating controls in Fornasini-Marchesini second local state space (FMSLSS) setting. A Lyapunov-based method to design an antiwindup gain of 2D discrete systems with saturating controls is established. Stability conditions allowing the design of antiwindup loops, in both local and global contexts have been derived. Numerical examples are provided to illustrate the applicability of the proposed method.

\section{Introduction}

An important problem which is always inherent to all dynamical systems is the presence of actuator saturation nonlinearities. Such nonlinearities may lead to performance degradation and even instability for feedback control systems. The stability analysis of the continuous as well as discrete time linear systems with saturating controls has been widely considered for one-dimensional (1D) systems [1-10]. The commonly used techniques to design controllers taking into account actuator saturation are (i) constrained model predictive control $[4,11]$, (ii) scheduled controllers [12], and (iii) antiwindup compensators [13-18]. Model predictive controllers find applications in chemical industries for the control of systems with saturations. Scheduled controllers also called piecewise linear controller or gain scheduling schemes are often used in aerospace industry. Antiwindup compensators are widely used in practice for the control systems with saturating actuators $[14,15]$. Design of antiwindup controllers can be carried out using linear design methods which explain its usefulness and popularity among control engineers. The actuator saturation problem is tackled following the "antiwindup paradigm" which employs a twostep design procedure. The main idea here is to design a linear controller ignoring the saturation nonlinearities and then augment this controller with extra dynamics to minimize the adverse effects of saturation on the closed loop performance. Several results as well as design schemes on the antiwindup problem and compensation gain are formulated and the stability conditions have been mentioned for 1D systems [7-10, 14-18].

In the recent years, two-dimensional (2D) discrete systems have found various applications in many areas such as filtering, image processing, seismographic data processing, thermal processes, gas absorption, and water stream heating [19-22]. Mathematically, a 2D discrete system is represented by a set of difference equations with two space coordinates. The stability properties of 2D discrete systems described by Fornasini-Marchesini second local state space (FMSLSS) model [19] has been studied in [23-33]. Lyapunov-based sufficient conditions for the global asymptotic stability of linear FMSLSS model have been reported in [23-26]. The stabilization problem of 2D continuous time saturated systems by state feedback control has been considered in [34]. The stability analysis of 2D discrete systems with state saturation nonlinearities has been carried out in [24, 27-33]. However, to the best of the authors' knowledge, no previous 
work has considered the area of "antiwindup paradigm" for 2-D saturated systems.

Inspired by the results [9] for 1D discrete-time systems, this paper investigates the antiwindup problem for $2 \mathrm{D}$ discrete systems described by FMSLSS model with saturating controls. The paper aims at providing a technique to compute the antiwindup gain of the 2D dynamic compensator that ensures the stability of the overall closed loop system in local as well as global context. Utilizing the sector description of saturation nonlinearities and 2D quadratic Lyapunov function, linear-matrix-inequality- (LMI-) based stability conditions are obtained.

The paper is organized as follows. In Section 2, the problem considered has been stated. The necessary concepts required in the paper have also been provided. LMI-based criteria for the stability of closed loop systems are developed in Section 3. In Section 4, the applicability of the presented approach has been demonstrated with the help of numerical examples. Section 5 presents the applicability of the designed antiwindup controller.

\section{Problem Statement}

Consider the 2D discrete system described by FMSLSS model [19]

$$
\begin{aligned}
\mathbf{x}(i+1, j+1)= & \mathbf{A}_{1} \mathbf{x}(i+1, j)+\mathbf{A}_{2} \mathbf{x}(i, j+1) \\
& +\mathbf{B}_{1} \mathbf{u}(i+1, j)+\mathbf{B}_{2} \mathbf{u}(i, j+1), \\
\mathbf{y}(i, j)= & \mathbf{C} \mathbf{x}(i, j),
\end{aligned}
$$

where $i \in Z_{+}, j \in Z_{+}$, and $Z_{+}$denotes the set of nonnegative integers. The $\mathbf{x}(i, j) \in \mathfrak{R}^{n}$ is a state vector, $\mathbf{u}(i, j) \in \mathbb{R}^{m}$ is an input vector, and $\mathbf{y}(i, j) \in \mathfrak{R}^{p}$ is an output vector. The matrices $\mathbf{A}_{k} \in \mathfrak{R}^{n \times n}, \mathbf{B}_{k} \in \mathfrak{R}^{n \times m}(k=1,2)$, and $\mathbf{C} \in$ $\mathfrak{R}^{p \times n}$ are known constant matrices representing a nominal plant.

Let a linear 2D dynamic compensator which stabilizes system (1) and meets the desired performance specifications in the absence of actuator saturation be given by

$$
\begin{aligned}
\mathbf{x}_{c}(i+1, j+1)= & \mathbf{A}_{c 1} \mathbf{x}_{c}(i+1, j)+\mathbf{A}_{c 2} \mathbf{x}_{c}(i, j+1) \\
& +\mathbf{B}_{c 1} \mathbf{u}_{c}(i+1, j)+\mathbf{B}_{c 2} \mathbf{u}_{c}(i, j+1), \\
\mathbf{v}_{c}(i, j)= & \mathbf{C}_{c} \mathbf{x}_{c}(i, j)+\mathbf{D}_{c} \mathbf{u}_{c}(i, j),
\end{aligned}
$$

where $\mathbf{x}_{c}(i, j) \in \boldsymbol{R}^{n_{c}}$ is a controller state vector, $\mathbf{u}_{c}(i, j)=$ $\mathbf{y}(i, j) \in \mathfrak{R}^{p}$ is a controller input vector, and $\mathbf{v}_{c}(i, j) \in \mathfrak{R}^{m}$ is a controller output vector. The matrices $\mathbf{A}_{c k} \in \mathfrak{R}^{n_{c} \times n_{c}}, \mathbf{B}_{c k} \in$ $\mathfrak{R}^{n_{c} \times p}(k=1,2), \mathbf{C}_{c} \in \mathfrak{R}^{m \times n_{c}}$, and $\mathbf{D}_{c} \in \mathfrak{R}^{m \times p}$ are constant matrices of appropriate dimensions.

Suppose that the input vector $\mathbf{u}(i, j)$ is subject to amplitude limitations defined as

$$
-u_{0(l)} \leq u_{(l)}(i, j) \leq u_{0(l)}
$$

where $u_{0(l)}>0, l=1, \ldots, m$, denote the control amplitude bounds. Consequently, the actual control signal injected to the system (1) is a saturated one given by

$$
\mathbf{u}(i, j)=\operatorname{sat}\left(\mathbf{v}_{c}(i, j)\right)=\operatorname{sat}\left[\mathbf{C}_{c} \mathbf{x}_{c}(i, j)+\mathbf{D}_{c} \mathbf{u}_{c}(i, j)\right] .
$$

The saturation nonlinearities characterized by

$$
\text { sat }\left(v_{c}(i, j)\right)_{(l)}= \begin{cases}-u_{0(l)}, & \text { if } v_{c(l)}<-u_{0(l)}, \\ v_{c(l)}, & \text { if }-u_{0(l)} \leq v_{c(l)} \leq u_{0(l)}, \\ u_{0(l)}, & \text { if } v_{c(l)}>u_{0(l)},\end{cases}
$$

where $l=1, \ldots, m$, are under consideration.

The actuator saturation causes windup of the controller and to mitigate its effect an antiwindup compensation term is added to the controller. A 2D antiwindup compensator involves adding a correction term of the form $\mathbf{E}_{c}\left[\operatorname{sat}\left(\mathbf{v}_{c}(i, j)\right)-\mathbf{v}_{c}(i, j)\right]$. The modified compensator has the form

$$
\begin{aligned}
\mathbf{x}(i+1, j+1)= & \mathbf{A}_{1} \mathbf{x}(i+1, j)+\mathbf{A}_{2} \mathbf{x}(i, j+1) \\
& +\mathbf{B}_{1} \operatorname{sat}\left(\mathbf{v}_{c}(i+1, j)\right) \\
& +\mathbf{B}_{2} \operatorname{sat}\left(\mathbf{v}_{c}(i, j+1)\right) \\
\mathbf{y}(i, j)= & \mathbf{C x}(i, j) \\
\mathbf{x}_{c}(i+1, j+1)= & \mathbf{A}_{c 1} \mathbf{x}_{c}(i+1, j)+\mathbf{A}_{c 2} \mathbf{x}_{c}(i, j+1) \\
& +\mathbf{B}_{c 1} \mathbf{C x}(i+1, j)+\mathbf{B}_{c 2} \mathbf{C x}(i, j+1) \\
& +\mathbf{E}_{c 1}\left[\operatorname{sat}\left(\mathbf{v}_{c}(i+1, j)\right)-\mathbf{v}_{c}(i+1, j)\right] \\
& +\mathbf{E}_{c 2}\left[\operatorname{sat}\left(\mathbf{v}_{c}(i, j+1)\right)-\mathbf{v}_{c}(i, j+1)\right] \\
\mathbf{v}_{c}(i, j)= & \mathbf{C}_{c} \mathbf{x}_{c}(i, j)+\mathbf{D}_{c} \mathbf{C x}(i, j)
\end{aligned}
$$

Let

$$
\psi\left(\mathbf{v}_{c}(i, j)\right)=\mathbf{v}_{c}(i, j)-\operatorname{sat}\left(\mathbf{v}_{c}(i, j)\right) .
$$

Substituting (7) into (6) we obtain

$$
\begin{aligned}
\mathbf{x}(i+1, j+1)= & \mathbf{A}_{1} \mathbf{x}(i+1, j)+\mathbf{A}_{2} \mathbf{x}(i, j+1) \\
& +\mathbf{B}_{1}\left[\left(\mathbf{v}_{c}(i+1, j)\right)-\psi\left(\mathbf{v}_{c}(i+1, j)\right)\right] \\
& +\mathbf{B}_{2}\left[\mathbf{v}_{c}(i, j+1)-\psi\left(\mathbf{v}_{c}(i, j+1)\right)\right] \\
= & \mathbf{A}_{1} \mathbf{x}(i+1, j)+\mathbf{A}_{2} \mathbf{x}(i, j+1) \\
& +\mathbf{B}_{1}\left[\mathbf{C}_{c} \mathbf{x}_{c}(i+1, j)+\mathbf{D}_{c} \mathbf{C} \mathbf{x}(i+1, j)\right. \\
& \left.-\psi\left(\mathbf{v}_{c}(i+1, j)\right)\right] \\
& +\mathbf{B}_{2}\left[\mathbf{C}_{c} \mathbf{x}_{c}(i, j+1)+\mathbf{D}_{c} \mathbf{C} \mathbf{x}(i, j+1)\right. \\
& \left.-\psi\left(\mathbf{v}_{c}(i, j+1)\right)\right]
\end{aligned}
$$




$$
\begin{aligned}
= & \mathbf{A}_{1} \mathbf{x}(i+1, j)+\mathbf{A}_{2} \mathbf{x}(i, j+1) \\
& +\mathbf{B}_{1}\left[\mathbf{C}_{c} \mathbf{x}_{c}(i+1, j)+\mathbf{D}_{c} \mathbf{C} \mathbf{x}(i+1, j)\right] \\
& -\mathbf{B}_{1} \psi\left[\mathbf{C}_{c} \mathbf{x}_{c}(i+1, j)+\mathbf{D}_{c} \mathbf{C} \mathbf{x}(i+1, j)\right] \\
& +\mathbf{B}_{2}\left[\mathbf{C}_{c} \mathbf{x}_{c}(i, j+1)+\mathbf{D}_{c} \mathbf{C} \mathbf{x}(i, j+1)\right] \\
& -\mathbf{B}_{2} \psi\left[\mathbf{C}_{c} \mathbf{x}_{c}(i, j+1)+\mathbf{D}_{c} \mathbf{C} \mathbf{x}(i, j+1)\right], \\
\mathbf{x}_{c}(i+1, j+1)= & \mathbf{A}_{c 1} \mathbf{x}_{c}(i+1, j)+\mathbf{A}_{c 2} \mathbf{x}_{c}(i, j+1) \\
& +\mathbf{B}_{c 1} \mathbf{C} \mathbf{x}(i+1, j)+\mathbf{B}_{c 2} \mathbf{C x}(i, j+1) \\
& +\mathbf{E}_{c 1}\left[\mathbf{v}_{c}(i+1, j)-\psi\left(\mathbf{v}_{c}(i+1, j)\right)\right. \\
& \left.\quad-\mathbf{v}_{c}(i+1, j)\right] \\
& +\mathbf{E}_{c 2}\left[\mathbf{v}_{c}(i, j+1)-\psi\left(\mathbf{v}_{c}(i, j+1)\right)\right. \\
& \left.\quad-\mathbf{v}_{c}(i, j+1)\right] \\
= & \mathbf{A}_{c 1} \mathbf{x}_{c}(i+1, j)+\mathbf{A}_{c 2} \mathbf{x}_{c}(i, j+1) \\
& +\mathbf{B}_{c 1} \mathbf{C} \mathbf{x}(i+1, j)+\mathbf{B}_{c 2} \mathbf{C x}(i, j+1) \\
& -\mathbf{E}_{c 1} \psi\left[\mathbf{C}_{c} \mathbf{x}_{c}(i+1, j)+\mathbf{D}_{c} \mathbf{C x}(i+1, j)\right] \\
& -\mathbf{E}_{c 2} \psi\left[\mathbf{C}_{c} \mathbf{x}_{c}(i, j+1)+\mathbf{D}_{c} \mathbf{C x}(i, j+1)\right] .
\end{aligned}
$$

Define an extended state vector

$$
\boldsymbol{\xi}(i, j)=\left[\begin{array}{l}
\mathbf{x}(i, j) \\
\mathbf{x}_{c}(i, j)
\end{array}\right] \in \mathfrak{R}^{n+n_{c}} .
$$

Using (8) and (9), the closed loop system can be written as

$$
\begin{aligned}
\xi(i+1, j+1)= & \overline{\mathbf{A}}_{1} \xi(i+1, j)-\left(\overline{\mathbf{B}}_{1}+\mathbf{R E}_{c 1}\right) \psi(\mathbf{K} \xi(i+1, j)) \\
& +\overline{\mathbf{A}}_{2} \boldsymbol{\xi}(i, j+1) \\
& -\left(\overline{\mathbf{B}}_{2}+\mathbf{R E}_{c 2}\right) \psi(\mathbf{K} \xi(i, j+1))
\end{aligned}
$$

where

$$
\begin{aligned}
& \overline{\mathbf{A}}_{1}=\left[\begin{array}{cc}
\mathbf{A}_{1}+\mathbf{B}_{1} \mathbf{D}_{c} \mathbf{C} & \mathbf{B}_{1} \mathbf{C}_{c} \\
\mathbf{B}_{c 1} \mathbf{C} & \mathbf{A}_{c 1}
\end{array}\right], \\
& \overline{\mathbf{A}}_{2}=\left[\begin{array}{cc}
\mathbf{A}_{2}+\mathbf{B}_{2} \mathbf{D}_{c} \mathbf{C} & \mathbf{B}_{2} \mathbf{C}_{c} \\
\mathbf{B}_{c 2} \mathbf{C} & \mathbf{A}_{c 2}
\end{array}\right], \\
& \overline{\mathbf{B}}_{1}=\left[\begin{array}{c}
\mathbf{B}_{1} \\
\mathbf{0}
\end{array}\right], \quad \overline{\mathbf{B}}_{2}=\left[\begin{array}{c}
\mathbf{B}_{2} \\
\mathbf{0}
\end{array}\right], \\
& \mathbf{R}=\left[\begin{array}{c}
\mathbf{0} \\
\mathbf{I}_{n_{c}}
\end{array}\right], \quad \mathbf{K}=\left[\begin{array}{ll}
\mathbf{D}_{c} \mathbf{C} & \mathbf{C}_{c}
\end{array}\right]
\end{aligned}
$$

and $\mathbf{I}_{n_{c}}$ is the identity matrix of order $n_{c}$. It is assumed [27$34]$ that the system has a finite set of initial conditions, that is, there exist two positive integers $h_{1}$ and $h_{2}$ such that

$$
\begin{aligned}
& \xi(i, 0)=\mathbf{0}, \quad i \geq h_{1} ; \\
& \xi(0, j)=\mathbf{0}, \quad j \geq h_{2} .
\end{aligned}
$$

The aim of this paper is to determine the antiwindup compensator gain matrix $\left[\begin{array}{lll}\mathbf{E}_{c 1} & \mathbf{E}_{c 2}\end{array}\right]$ and an associated region of asymptotic stability of the closed loop system (10a)-(10b) for a given set of admissible initial states.

In the following section, we will establish stability condition for system given by (10a)-(10b) in both local and global contexts.

\section{Main Results}

Consider a block diagonal matrix $\mathbf{G} \in \mathfrak{R}^{2 m \times 2\left(n+n_{c}\right)}$ such that

$$
\mathbf{G}=\left[\begin{array}{cc}
\mathbf{G}_{1} & \mathbf{0} \\
\mathbf{0} & \mathbf{G}_{2}
\end{array}\right],
$$

where $\mathbf{G}_{1} \in \mathfrak{R}^{m \times\left(n+n_{c}\right)}, \mathbf{G}_{2} \in \mathfrak{R}^{m \times\left(n+n_{c}\right)}$ and define a polyhedral set:

$$
\begin{gathered}
\ell \triangleq\left\{\boldsymbol{\xi} \in \mathfrak{R}^{\left(n+n_{c}\right)} ;-u_{0(l)} \leq\left(\mathbf{K}_{(l)}-\mathbf{G}_{1(l)}\right) \boldsymbol{\xi}(i+1, j)\right. \\
\leq u_{0(l)},-u_{0(l)} \leq\left(\mathbf{K}_{(l)}-\mathbf{G}_{2(l)}\right) \boldsymbol{\xi}(i, j+1) \leq u_{0(l)}, \\
\quad l=1,2, \ldots, m\} .
\end{gathered}
$$

Now, we have the following lemma.

Lemma 1. If $\xi \in \ell$ then

$$
\begin{aligned}
\delta= & {\left[\begin{array}{l}
\psi(\mathbf{K} \xi(i+1, j)) \\
\psi(\mathbf{K} \xi(i, j+1))
\end{array}\right]^{T} } \\
& \times \mathbf{D}\left\{\left[\begin{array}{l}
\psi(\mathbf{K} \xi(i+1, j)) \\
\psi(\mathbf{K} \xi(i, j+1))
\end{array}\right]-\mathbf{G}\left[\begin{array}{l}
\boldsymbol{\xi}(i+1, j) \\
\boldsymbol{\xi}(i, j+1)
\end{array}\right]\right\} \leq 0,
\end{aligned}
$$

where $\mathbf{D}$ is positive definite block diagonal matrix and " $T$ " denotes the transpose.

Proof. Observe that, (14) can be expressed as

$$
\begin{aligned}
\delta= & \psi^{T}(\mathbf{K} \xi(i+1, j)) \\
& \times \mathbf{D}_{1}\left\{\psi(\mathbf{K} \xi(i+1, j))-\mathbf{G}_{1} \xi(i+1, j)\right\} \\
& +\psi^{T}(\mathbf{K} \xi(i, j+1)) \\
& \times \mathbf{D}_{2}\left\{\psi(\mathbf{K} \xi(i, j+1))-\mathbf{G}_{2} \xi(i, j+1)\right\} \leq 0,
\end{aligned}
$$


where $\mathbf{D}_{1} \in \mathfrak{R}^{m \times m}, \mathbf{D}_{2} \in \mathfrak{R}^{m \times m}$ are positive definite diagonal matrices and $\mathbf{D}=\left[\begin{array}{cc}\mathbf{D}_{1} & 0 \\ 0 & \mathbf{D}_{2}\end{array}\right]$. Following the proof of [9, Lemma 1], it can be shown that both terms of the left hand side of (15) are nonpositive. This completes the proof.

The main result of the paper may be stated as follows.

Theorem 1. Suppose there exists a positive definite symmetric matrix $\mathbf{W} \in \mathfrak{R}^{\left(n+n_{c}\right) \times\left(n+n_{c}\right)}$, a matrix $\mathbf{Z} \in \mathfrak{R}^{n_{c} \times 2 m}$, a matrix $\mathbf{Y} \in \mathfrak{R}^{2 m \times 2\left(n+n_{c}\right)}$, a scalar $0<\alpha<1$, and a diagonal positive definite matrix $\mathbf{S} \in \mathfrak{R}^{2 m \times 2 m}$ satisfying the following LMIs:

$$
\begin{aligned}
& {\left[\begin{array}{ccc}
\overline{\mathbf{P}}^{-1} & -\mathbf{Y}^{T} & -\overline{\mathbf{P}}^{-1} \overline{\mathbf{A}}^{T} \\
-\mathbf{Y} & 2 \mathbf{S} & (\overline{\mathbf{B}} \mathbf{S}+\mathbf{R Z})^{T} \\
-\overline{\mathbf{A}} \overline{\mathbf{P}}^{-1} & (\overline{\mathbf{B}} \mathbf{S}+\mathbf{R Z}) & \mathbf{W}
\end{array}\right]>\mathbf{0},} \\
& {\left[\begin{array}{cc}
\alpha^{-1} \mathbf{W} & \alpha^{-1} \mathbf{W} \mathbf{K}_{(l)}^{T}-\mathbf{Y}_{1(l)}^{T} \\
\alpha^{-1} \mathbf{K}_{(l)} \mathbf{W}-\mathbf{Y}_{1(l)} & u_{0(l)}^{2}
\end{array}\right] \geq \mathbf{0}, \quad l=1,2, \ldots, m,} \\
& {\left[\begin{array}{cc}
(1-\alpha)^{-1} \mathbf{W} & (1-\alpha)^{-1} \mathbf{W} \mathbf{K}_{(l)}^{T}-\mathbf{Y}_{2(l)}^{T} \\
(1-\alpha)^{-1} \mathbf{K}_{(l)} \mathbf{W}-\mathbf{Y}_{2(l)} & u_{0(l)}^{2}
\end{array}\right] \geq \mathbf{0},}
\end{aligned}
$$

where

$$
\overline{\mathbf{P}}^{-1}=\left[\begin{array}{cc}
\alpha^{-1} \mathbf{W} & \mathbf{0} \\
\mathbf{0} & (1-\alpha)^{-1} \mathbf{W}
\end{array}\right] .
$$

Then for the gain matrix $\mathbf{E}_{c}=\mathbf{Z S}^{-1}$ the ellipsoid $\varepsilon(\mathbf{P})=$ $\left\{\boldsymbol{\xi} \in \mathfrak{R}^{n+n_{c}} ; \boldsymbol{\xi}^{T} \mathbf{P} \boldsymbol{\xi} \leq \min (1 / \alpha, 1 /(1-\alpha))\right\}$ with $\mathbf{P}=\mathbf{W}^{-1}$ is a region of asymptotic stability for system (10a)-(10b).

Proof. Consider a 2D quadratic Lyapunov function

$$
V(i+\eta, j+\tau)=\boldsymbol{\xi}^{T}(i+\eta, j+\tau) \mathbf{P} \boldsymbol{\xi}(i+\eta, j+\tau),
$$

where $\mathbf{P}=\mathbf{P}^{T}>\mathbf{0}$. Now, following [23] (see [24, 25] also), we define $\Delta V(i, j)$ as

$$
\begin{aligned}
\Delta V(i, j)= & \xi^{T}(i+1, j+1) \mathbf{P} \boldsymbol{\xi}(i+1, j+1) \\
& -\boldsymbol{\xi}^{T}(i+1, j) \alpha \mathbf{P} \boldsymbol{\xi}(i+1, j) \\
& -\xi^{T}(i, j+1)(1-\alpha) \mathbf{P} \boldsymbol{\xi}(i, j+1) .
\end{aligned}
$$

Using (10a)-(10b), (21) can be rearranged as

$\Delta V(i, j)$

$$
\begin{aligned}
= & {\left[\overline{\mathbf{A}}_{1} \boldsymbol{\xi}(i+1, j)-\left(\overline{\mathbf{B}}_{1}+\mathbf{R E}_{c 1}\right) \psi(\mathbf{K} \boldsymbol{\xi}(i+1, j))\right.} \\
& \left.+\overline{\mathbf{A}}_{2} \xi(i, j+1)-\left(\overline{\mathbf{B}}_{2}+\mathbf{R E}_{c 2}\right) \psi(\mathbf{K} \xi(i, j+1))\right]^{T} \mathbf{P}
\end{aligned}
$$

$$
\begin{aligned}
& \times\left[\overline{\mathbf{A}}_{1} \boldsymbol{\xi}(i+1, j)-\left(\overline{\mathbf{B}}_{1}+\mathbf{R E}_{c 1}\right) \psi(\mathbf{K} \boldsymbol{\xi}(i+1, j))\right. \\
& \left.+\overline{\mathbf{A}}_{2} \boldsymbol{\xi}(i, j+1)-\left(\overline{\mathbf{B}}_{2}+\mathbf{R E}_{c 2}\right) \boldsymbol{\psi}(\mathbf{K} \boldsymbol{\xi}(i, j+1))\right] \\
& -\boldsymbol{\xi}^{T}(i+1, j) \alpha \mathbf{P} \boldsymbol{\xi}(i+1, j) \\
& -\boldsymbol{\xi}^{T}(i, j+1)(1-\alpha) \mathbf{P} \boldsymbol{\xi}(i, j+1) \\
& =\boldsymbol{\xi}^{T}(i+1, j) \overline{\mathbf{A}}_{1}^{T} \mathbf{P} \overline{\mathbf{A}}_{1} \boldsymbol{\xi}(i+1, j) \\
& -\boldsymbol{\xi}^{T}(i+1, j) \overline{\mathbf{A}}_{1}^{T} \mathbf{P}\left(\overline{\mathbf{B}}_{1}+\mathbf{R E}_{c 1}\right) \psi(\mathbf{K} \boldsymbol{\xi}(i+1, j)) \\
& -\boldsymbol{\psi}^{T}(\mathbf{K} \boldsymbol{\xi}(i+1, j))\left(\overline{\mathbf{B}}_{1}+\mathbf{R E}_{c 1}\right)^{T} \mathbf{P} \overline{\mathbf{A}}_{1} \boldsymbol{\xi}(i+1, j) \\
& +\boldsymbol{\psi}^{T}(\mathbf{K} \boldsymbol{\xi}(i+1, j))\left(\overline{\mathbf{B}}_{1}+\mathbf{R E}_{c 1}\right)^{T} \\
& \times \mathbf{P}\left(\overline{\mathbf{B}}_{1}+\mathbf{R E}_{c 1}\right) \psi(\mathbf{K} \boldsymbol{\xi}(i+1, j)) \\
& +\boldsymbol{\xi}^{T}(i+1, j) \overline{\mathbf{A}}_{1}^{T} \mathbf{P} \overline{\mathbf{A}}_{2} \boldsymbol{\xi}(i, j+1) \\
& -\boldsymbol{\xi}^{T}(i+1, j) \overline{\mathbf{A}}_{1}^{T} \mathbf{P}\left(\overline{\mathbf{B}}_{2}+\mathbf{R E}_{c 2}\right) \psi(\mathbf{K} \boldsymbol{\xi}(i, j+1)) \\
& -\boldsymbol{\psi}^{T}(\mathbf{K} \boldsymbol{\xi}(i+1, j))\left(\overline{\mathbf{B}}_{1}+\mathbf{R E}_{c 1}\right)^{T} \mathbf{P} \overline{\mathbf{A}}_{2} \boldsymbol{\xi}(i, j+1) \\
& +\boldsymbol{\psi}^{T}(\mathbf{K} \boldsymbol{\xi}(i+1, j))\left(\overline{\mathbf{B}}_{1}+\mathbf{R E}_{c 1}\right)^{T} \\
& \times \mathbf{P}\left(\overline{\mathbf{B}}_{2}+\mathbf{R E}_{c 2}\right) \psi(\mathbf{K} \boldsymbol{\xi}(i, j+1)) \\
& +\boldsymbol{\xi}^{T}(i, j+1) \overline{\mathbf{A}}_{2}^{T} \mathbf{P} \overline{\mathbf{A}}_{1} \boldsymbol{\xi}(i+1, j) \\
& -\boldsymbol{\xi}^{T}(i, j+1) \overline{\mathbf{A}}_{2}^{T} \mathbf{P}\left(\overline{\mathbf{B}}_{1}+\mathbf{R E}_{c 1}\right) \psi(\mathbf{K} \boldsymbol{\xi}(i+1, j)) \\
& +\boldsymbol{\xi}^{T}(i, j+1) \overline{\mathbf{A}}_{2}^{T} \mathbf{P} \overline{\mathbf{A}}_{2} \boldsymbol{\xi}(i, j+1) \\
& -\boldsymbol{\xi}^{T}(i, j+1) \overline{\mathbf{A}}_{2}^{T} \mathbf{P}\left(\overline{\mathbf{B}}_{2}+\mathbf{R E}_{c 2}\right) \psi(\mathbf{K} \boldsymbol{\xi}(i, j+1)) \\
& -\boldsymbol{\psi}^{T}(\mathbf{K} \boldsymbol{\xi}(i, j+1))\left(\overline{\mathbf{B}}_{2}+\mathbf{R E}_{c 2}\right)^{T} \mathbf{P} \overline{\mathbf{A}}_{1} \boldsymbol{\xi}(i+1, j) \\
& +\boldsymbol{\psi}^{T}(\mathbf{K} \boldsymbol{\xi}(i, j+1))\left(\overline{\mathbf{B}}_{2}+\mathbf{R E}_{c 2}\right)^{T} \\
& \times \mathbf{P}\left(\overline{\mathbf{B}}_{1}+\mathbf{R E}_{c 1}\right) \psi(\mathbf{K} \boldsymbol{\xi}(i+1, j)) \\
& -\boldsymbol{\psi}^{T}(\mathbf{K} \boldsymbol{\xi}(i, j+1))\left(\overline{\mathbf{B}}_{2}+\mathbf{R E}_{c 2}\right)^{T} \mathbf{P} \overline{\mathbf{A}}_{2} \boldsymbol{\xi}(i, j+1) \\
& +\boldsymbol{\psi}^{T}(\mathbf{K} \boldsymbol{\xi}(i, j+1))\left(\overline{\mathbf{B}}_{2}+\mathbf{R E}_{c 2}\right)^{T} \\
& \times \mathbf{P}\left(\overline{\mathbf{B}}_{2}+\mathbf{R E}_{c 2}\right) \psi(\mathbf{K} \boldsymbol{\xi}(i, j+1)) \\
& -\boldsymbol{\xi}^{T}(i+1, j) \alpha \mathbf{P} \boldsymbol{\xi}(i+1, j) \\
& -\boldsymbol{\xi}^{T}(i, j+1)(1-\alpha) \mathbf{P} \boldsymbol{\xi}(i, j+1) .
\end{aligned}
$$

Adding to and subtracting from (22) the quantity $2 \delta$ (see (15)), we obtain 


$$
\begin{aligned}
& \Delta V(i, j)=-\left[\begin{array}{llll}
\boldsymbol{\xi}^{T}(i+1, j) & \boldsymbol{\xi}^{T}(i, j+1) & \boldsymbol{\psi}^{T}(\mathbf{K} \boldsymbol{\xi}(i+1, j)) & \boldsymbol{\psi}^{T}(\mathbf{K} \boldsymbol{\xi}(i, j+1))
\end{array}\right] \\
& \times\left[\begin{array}{cc}
\alpha \mathbf{P}-\overline{\mathbf{A}}_{1}^{T} \mathbf{P} \overline{\mathbf{A}}_{1} & -\overline{\mathbf{A}}_{1}^{T} \mathbf{P} \overline{\mathbf{A}}_{2} \\
-\overline{\mathbf{A}}_{2}^{T} \mathbf{P} \overline{\mathbf{A}}_{1} & (1-\alpha) \mathbf{P}-\overline{\mathbf{A}}_{2}^{T} \mathbf{P} \overline{\mathbf{A}}_{2} \\
\left(\overline{\mathbf{B}}_{1}+\mathbf{R E}_{c 1}\right)^{T} \mathbf{P} \overline{\mathbf{A}}_{1}-\mathbf{D}_{1} \mathbf{G}_{1} & \left(\overline{\mathbf{B}}_{1}+\mathbf{R} \mathbf{E}_{c 1}\right)^{T} \mathbf{P} \overline{\mathbf{A}}_{2} \\
\left(\overline{\mathbf{B}}_{2}+\mathbf{R} \mathbf{E}_{c 2}\right)^{T} \mathbf{P} \overline{\mathbf{A}}_{1} & \left(\overline{\mathbf{B}}_{2}+\mathbf{R E}_{c 2}\right)^{T} \mathbf{P} \overline{\mathbf{A}}_{2}-\mathbf{D}_{2} \mathbf{G}_{2}
\end{array}\right. \\
& \overline{\mathbf{A}}_{1}^{T} \mathbf{P}\left(\overline{\mathbf{B}}_{1}+\mathbf{R E}_{c 1}\right)-\mathbf{G}_{1}^{T} \mathbf{D}_{1} \quad \overline{\mathbf{A}}_{1}^{T} \mathbf{P}\left(\overline{\mathbf{B}}_{2}+\mathbf{R} \mathbf{E}_{c 2}\right) \\
& \overline{\mathbf{A}}_{2}^{T} \mathbf{P}\left(\overline{\mathbf{B}}_{1}+\mathbf{R} \mathbf{E}_{c 1}\right) \\
& \overline{\mathbf{A}}_{2}^{T} \mathbf{P}\left(\overline{\mathbf{B}}_{2}+\mathbf{R} \mathbf{E}_{c 2}\right)-\mathbf{G}_{2}^{T} \mathbf{D}_{2} \\
& 2 \mathbf{D}_{1}-\left(\overline{\mathbf{B}}_{1}+\mathbf{R} \mathbf{E}_{c 1}\right)^{T} \mathbf{P}\left(\overline{\mathbf{B}}_{1}+\mathbf{R E}_{c 1}\right) \quad-\left(\overline{\mathbf{B}}_{1}+\mathbf{R} \mathbf{E}_{c 1}\right)^{T} \mathbf{P}\left(\overline{\mathbf{B}}_{2}+\mathbf{R} \mathbf{E}_{c 2}\right) \\
& \left.-\left(\overline{\mathbf{B}}_{2}+\mathbf{R E}_{c 2}\right)^{T} \mathbf{P}\left(\overline{\mathbf{B}}_{1}+\mathbf{R E}_{c 1}\right) \quad 2 \mathbf{D}_{2}-\left(\overline{\mathbf{B}}_{2}+\mathbf{R E}_{c 2}\right)^{T} \mathbf{P}\left(\overline{\mathbf{B}}_{2}+\mathbf{R E}_{c 2}\right)\right] \\
& \times\left[\begin{array}{c}
\boldsymbol{\xi}(i+1, j) \\
\boldsymbol{\xi}(i, j+1) \\
\psi(\mathbf{K} \boldsymbol{\xi}(i+1, j)) \\
\psi(\mathbf{K} \boldsymbol{\xi}(i, j+1))
\end{array}\right]+2 \delta
\end{aligned}
$$

Defining

$$
\begin{aligned}
\overline{\mathbf{A}} & =\left[\begin{array}{ll}
\overline{\mathbf{A}}_{1} & \overline{\mathbf{A}}_{2}
\end{array}\right], \\
\overline{\mathbf{B}} & =\left[\begin{array}{ll}
\overline{\mathbf{B}}_{1} & \overline{\mathbf{B}}_{2}
\end{array}\right], \\
\mathbf{E}_{c} & =\left[\begin{array}{ll}
\mathbf{E}_{c 1} & \mathbf{E}_{c 2}
\end{array}\right]
\end{aligned}
$$

equation (23) can be written as

$$
\begin{aligned}
\Delta V(i, j)=- & {\left[\begin{array}{c}
\boldsymbol{\xi}(i+1, j) \\
\boldsymbol{\xi}(i, j+1) \\
\psi(\mathbf{K} \boldsymbol{\xi}(i+1, j)) \\
\psi(\mathbf{K} \boldsymbol{\xi}(i, j+1))
\end{array}\right]^{T}\left[\begin{array}{ll}
\mathbf{X}_{1} & \mathbf{X}_{2} \\
\mathbf{X}_{2}^{T} & \mathbf{X}_{3}
\end{array}\right] } \\
& \times\left[\begin{array}{c}
\boldsymbol{\xi}(i+1, j) \\
\boldsymbol{\xi}(i, j+1) \\
\psi(\mathbf{K} \boldsymbol{\xi}(i+1, j)) \\
\psi(\mathbf{K} \boldsymbol{\xi}(i, j+1))
\end{array}\right]+2 \delta, \\
\mathbf{X}_{1}= & \overline{\mathbf{P}}-\overline{\mathbf{A}} \mathbf{P} \overline{\mathbf{A}}, \\
\mathbf{X}_{2}= & \overline{\mathbf{A}}^{T} \mathbf{P}\left(\overline{\mathbf{B}}+\mathbf{R E}_{c}\right)-\mathbf{G}{ }^{T} \mathbf{D}, \\
\mathbf{X}_{3}= & 2 \mathbf{D}-\left(\overline{\mathbf{B}}+\mathbf{R E}_{c}\right)^{T} \mathbf{P}\left(\overline{\mathbf{B}}+\mathbf{R E}_{c}\right),
\end{aligned}
$$

where $\overline{\mathbf{P}}$ is given by (19). From (25) and (15) it is clear that $\Delta V(i, j) \leq 0$ if

$$
\left[\begin{array}{ll}
\mathbf{X}_{1} & \mathbf{X}_{2} \\
\mathbf{X}_{2}^{T} & \mathbf{X}_{3}
\end{array}\right]>\mathbf{0}
$$

Before and after multiplying (26) by $\left[\begin{array}{cc}\overline{\mathbf{P}}^{-1} & 0 \\ 0 & \mathbf{D}^{-1}\end{array}\right]$ with $\mathbf{D}=\mathbf{S}^{-1}$, we obtain

$$
\begin{aligned}
& {\left[\begin{array}{cc}
\overline{\mathbf{P}}^{-1}-\overline{\mathbf{P}}^{-1} \overline{\mathbf{A}}^{T} \mathbf{P} \overline{\mathbf{A}} \overline{\mathbf{P}}^{-1} & \overline{\mathbf{P}}^{-1} \overline{\mathbf{A}}^{T} \mathbf{P}(\overline{\mathbf{B}} \mathbf{S}+\mathbf{R Z})-\overline{\mathbf{P}}^{-1} \mathbf{G}^{T} \\
(\overline{\mathbf{B}} \mathbf{S}+\mathbf{R Z})^{T} \mathbf{P} \overline{\mathbf{A}} \overline{\mathbf{P}}^{-1}-\mathbf{G} \overline{\mathbf{P}}^{-1} & 2 \mathbf{S}-(\overline{\mathbf{B}} \mathbf{S}+\mathbf{R Z})^{T} \mathbf{P}(\overline{\mathbf{B}} \mathbf{S}+\mathbf{R Z})
\end{array}\right]} \\
& \quad>0 .
\end{aligned}
$$

The equivalence of (27) and (16) trivially follows from Schur's complement.

Next, we will show that the satisfaction of (17) and (18) implies that the set $\varepsilon(\mathbf{P})=\left\{\boldsymbol{\xi} \in \mathbb{R}^{n+n_{c}} ; \boldsymbol{\xi}^{T} \mathbf{P} \boldsymbol{\xi} \leq\right.$ $\min (1 / \alpha, 1 /(1-\alpha))\}$ is included in the polyhedral set $\ell$ as defined in (13). It can be shown that $\left\{\boldsymbol{\xi} \in \mathfrak{R}^{n+n_{c}} ; \boldsymbol{\xi}^{T} \mathbf{P} \boldsymbol{\xi} \leq\right.$ $1 / \alpha\} \subset \ell$ is equivalent to [35]

$$
\alpha \mathbf{P}-\left(\mathbf{K}_{(l)}-\mathbf{G}_{1(l)}\right)^{T}\left(\mathbf{K}_{(l)}-\mathbf{G}_{1(l)}\right) u_{0}^{-2}(l) \geq \mathbf{0}, \quad l=1,2 \ldots m .
$$

Before and after multiplying (28) by $\alpha^{-1} \mathbf{P}^{-1}$ we get

$$
\begin{aligned}
& \alpha^{-1} \mathbf{P}^{-1}-\alpha^{-1} \mathbf{P}^{-1}\left(\mathbf{K}_{(l)}-\mathbf{G}_{1(l)}\right)^{T} \\
& \quad \times\left(\mathbf{K}_{(l)}-\mathbf{G}_{1(l)}\right) \alpha^{-1} \mathbf{P}^{-1} u_{0}^{-2}(l) \geq \mathbf{0} .
\end{aligned}
$$

By Schur's complement, (29) together with $\mathbf{G}_{1}=\alpha \mathbf{Y}_{1} \mathbf{P}$ and $\mathbf{P}=\mathbf{W}^{-1}$ leads to (17). Similarly, using (13) with $\mathbf{G}_{2}=$ $(1-\alpha) \mathbf{Y}_{2} \mathbf{P}$ and $\mathbf{P}=\mathbf{W}^{-1}$ it can be shown that $\{\boldsymbol{\xi} \in$ $\left.\mathfrak{R}^{n+n_{c}} ; \boldsymbol{\xi}^{T} \mathbf{P} \boldsymbol{\xi} \leq 1 /(1-\alpha)\right\} \subset \ell$ implies (18). This completes the proof.

Remark 1. Using Theorem 1, one can determine antiwindup gain matrix $\mathbf{E}_{c}$ in order to ensure the stability for a given 
region in the state space of $2 \mathrm{D}$ discrete systems with saturated inputs.

Remark 2. From (25) it is clear that $\Delta V(i, j) \leq 0$ for the system (10a)-(10b) with $\psi(\mathbf{K} \xi(i+1, j))=\psi(\mathbf{K} \xi(i, j+1))=$ $\mathbf{0}$ provided that $\overline{\mathbf{P}}-\overline{\mathbf{A}}^{T} \mathbf{P} \overline{\mathbf{A}}>\mathbf{0}$. Thus, the condition $\overline{\mathbf{P}}-$ $\overline{\mathbf{A}}^{T} \mathbf{P} \overline{\mathbf{A}}>\mathbf{0}$ (also known as Hinamoto's condition [23]) is a sufficient condition for the global asymptotic stability of $2 \mathrm{D}$ linear FMSLSS model.

Remark 3. It may be mentioned that several previous works [24, 27-33] deal with the problem of global asymptotic stability of digital filters with state saturation. The nonlinearities considered in [24, 27-33] occur due to the implementation of the system using finite wordlength. In contrast, the present paper tackles the problem of stability of a $2 \mathrm{D}$ system in presence of the actuator saturation nonlinearity.

As a direct consequence of Theorem 1, we have the following result for the global exponential stability of system (10a) and (10b).

Corollary 1. Suppose there exist a positive definite symmetric matrix $\mathbf{W} \in \mathfrak{R}^{\left(n+n_{c}\right) \times\left(n+n_{c}\right)}$, a diagonal positive definite matrix $\mathbf{S} \in \mathfrak{R}^{2 m \times 2 m}$, and a matrix $\mathbf{Z} \in \mathfrak{R}^{n_{c} \times 2 m}$ such that

$$
\left[\begin{array}{ccc}
\overline{\mathbf{P}}^{-1} & -\overline{\mathbf{P}}^{-1} \overline{\mathbf{K}}^{T} & -\overline{\mathbf{P}}^{-1} \overline{\mathbf{A}}^{T} \\
-\overline{\mathbf{K}} \overline{\mathbf{P}}^{-1} & 2 \mathbf{S} & (\overline{\mathbf{B}} \mathbf{S}+\mathbf{R Z})^{T} \\
-\overline{\mathbf{A}} \overline{\mathbf{P}}^{-1} & (\overline{\mathbf{B}} \mathbf{S}+\mathbf{R Z}) & \mathbf{W}
\end{array}\right]>\mathbf{0},
$$

where $\overline{\mathbf{P}}^{-1}$ is defined in (19). Then, for $\mathbf{E}_{c}=\mathbf{Z S ^ { - 1 }}$, the origin of system (10a)-(10b) is globally stable.

Proof. Choosing

$$
\mathbf{G}_{1}=\mathbf{G}_{2}=\mathbf{K}
$$

one can see that (13) is automatically met for all $\xi \in \mathfrak{R}^{n+n_{c}}$. Consequently, (14) is also satisfied for all $\xi \in \mathfrak{R}^{n+n_{c}}$. Now, substituting (31) into (16) we obtain the global exponential stability condition (30). This completes the proof.

Remark 4. Note that, unlike (16)-(19), (30) is independent of $u_{0(l)}, l=1,2, \ldots m$. In other words, one is not required to explicitly know the values of control amplitude bounds when dealing with Corollary 1.

Remark 5. It should be observed that for a given $\alpha(0<\alpha<$ $1)$ the matrix inequality (16)-(18) is linear in variables $\mathbf{W}, \mathbf{S}$, $\mathbf{Y}$, and $\mathbf{Z}$. Hence, it can be solved efficiently by employing the MATLAB LMI Toolbox $[35,36]$.

\section{Numerical Examples}

To illustrate the applicability of the presented results, we now consider the following examples.
Example 1. Consider a closed loop 2D system represented by (10a)-(10b) with the following parameters:

$$
\begin{aligned}
& \mathbf{A}_{1}=\left[\begin{array}{cc}
0.05 & 0.08 \\
0.2 & 0.3
\end{array}\right], \mathbf{A}_{2}=\left[\begin{array}{ll}
0.1 & 0.0 \\
0.0 & 0.1
\end{array}\right], \\
& \mathbf{B}_{1}=\left[\begin{array}{c}
0 \\
0.01
\end{array}\right], \mathbf{B}_{2}=\left[\begin{array}{c}
0 \\
0.01
\end{array}\right], \\
& \mathbf{A}_{c 1}=[0.1], \mathbf{A}_{c 2}=[0.1], \\
& \mathbf{B}_{c 1}=[0.01], \mathbf{B}_{c 2}=[0.01], \\
& \mathbf{C}=\left[\begin{array}{ll}
1 & 0
\end{array}\right], \mathbf{C}_{c}=[0.1] \\
& \mathbf{D}_{c}=[10]
\end{aligned}
$$

Using MATLAB LMI toolbox [36] and choosing $\alpha=0.5$, it is found that (30) is feasible for the following values of unknown parameters:

$$
\begin{aligned}
& \mathbf{W}=\left[\begin{array}{ccc}
27.6 & 1.0 & -14.5 \\
1.0 & 977.7 & -3.8 \\
-14.5 & -3.8 & 1503.1
\end{array}\right] \\
& \mathbf{S}=\left[\begin{array}{cc}
4016.8 & 0 \\
0 & 4002.7
\end{array}\right] \text {, } \\
& Z=\left[\begin{array}{ll}
-64.8397 & -92.7441
\end{array}\right] \text {. }
\end{aligned}
$$

In this case, the gain matrix $\mathbf{E}_{c}=\mathbf{Z S}^{-1}$ is given by

$$
\mathbf{E}_{c}=\left[\begin{array}{ll}
-0.0161 & -0.0232
\end{array}\right]
$$

Therefore, according to Corollary 1 , the system under consideration is globally stable.

Example 2. Consider a closed loop system described by (10a) and (10b) with

$$
\begin{array}{cc}
\mathbf{A}_{1}=\left[\begin{array}{cc}
0.6 & 0.08 \\
0.2 & 0.5
\end{array}\right], & \mathbf{A}_{2}=\left[\begin{array}{ll}
0.3 & 0.0 \\
0.0 & 0.1
\end{array}\right], \\
\mathbf{B}_{1}=\left[\begin{array}{c}
0 \\
0.01
\end{array}\right], & \mathbf{B}_{2}=\left[\begin{array}{c}
0 \\
0.01
\end{array}\right], \\
\mathbf{A}_{c 1}=[0.1], & \mathbf{A}_{c 2}=[0.1], \\
\mathbf{B}_{c 1}=[0.01], & \mathbf{B}_{c 2}=[0.01], \\
\mathbf{C}_{c}=[0.1], & \mathbf{C}=\left[\begin{array}{ll}
1 & 0
\end{array}\right], \\
\mathbf{D}_{c}=[10] .
\end{array}
$$


Using MATLAB LMI toolbox [36] and choosing $\alpha=0.5$ and the control bound $u_{0(1)}=1$, it is verified that (16)-(18) are feasible for the following values of unknown parameters

$$
\begin{gathered}
\mathbf{W}=\left[\begin{array}{ccc}
1.0 & 0.0 & -21 \\
0.0 & 1.0 & -38 \\
-21 & -38 & 14436
\end{array}\right], \\
\mathbf{S}=\left[\begin{array}{cc}
1511.5 & 0 \\
0 & 1255.2
\end{array}\right], \\
\mathbf{Z}=\left[\begin{array}{ll}
-4601.4 & -3843.7
\end{array}\right] .
\end{gathered}
$$

In this example, the gain matrix $\mathbf{E}_{c}=\mathbf{Z S}^{-1}$ is obtained as

$$
\mathbf{E}_{c}=\left[\begin{array}{ll}
-3.0442 & -3.0621
\end{array}\right] \text {. }
$$

Therefore, Theorem 1 assures that the system under consideration is asymptotically stable in the region given by the ellipsoid $\varepsilon(\mathbf{P})=\left\{\boldsymbol{\xi} \in \mathfrak{R}^{n+n_{c}} ; \boldsymbol{\xi}^{T} \mathbf{P} \boldsymbol{\xi} \leq 2\right\}$.

\section{Application to the Antiwindup Control of Dynamical Processes Described by the Darboux Equation}

The design method of antiwindup controller given in Section 3 can be applied to the control of several dynamical processes. It is known that in real world situations, some dynamical processes in water steam heating, gas absorption, and air drying can be described by the Darboux equation [37-39]. In this section, we shall illustrate the applicability of our proposed method (Theorem 1) in anti-windup control of processes in a Darboux equation.

Consider the Darboux equation [37-39] given by

$$
\begin{aligned}
\frac{\partial^{2} s(x, t)}{\partial x \partial t} & =a_{1} \frac{\partial s(x, t)}{\partial t}+a_{2} \frac{\partial s(x, t)}{\partial x}+a_{0} s(x, t)+b f(x, t), \\
y(x, t) & =c_{1}\left[\frac{\partial s(x, t)}{\partial t}-a_{2} s(x, t)\right]+c_{2} s(x, t),
\end{aligned}
$$

with the initial conditions:

$$
s(x, 0)=p(x), \quad s(0, t)=q(t),
$$

where $s(x, t)$ is an unknown function at space $x \in\left[0, x_{f}\right]$ and time $t \in[0, \infty] ; f(x, t)$ is the input function; $y(x, t)$ is the measured output; $a_{1}, a_{2}, a_{0}, b, c_{1}$, and $c_{2}$ are real constants.

Define

$$
r(x, t)=\frac{\partial s(x, t)}{\partial t}-a_{2} s(x, t)
$$

then (38a) can be transformed into an equivalent system of first-order differential equation of the form

$$
\left[\begin{array}{l}
\frac{\partial r(x, t)}{\partial x} \\
\frac{\partial s(x, t)}{\partial t}
\end{array}\right]=\left[\begin{array}{cc}
a_{1} & a_{1} a_{2}+a_{0} \\
1 & a_{2}
\end{array}\right]\left[\begin{array}{l}
r(x, t) \\
s(x, t)
\end{array}\right]+\left[\begin{array}{l}
b \\
0
\end{array}\right] f(x, t) .
$$

It follows from (40) that

$$
r(0, t)=\left.\frac{\partial s(x, t)}{\partial t}\right|_{x=0}-a_{2} s(0, t)=\frac{d q(t)}{d t}-a_{2} q(t)=z(t) .
$$

Taking $r(i, j)=r(i \Delta x, j \Delta t), s(i, j)=s(i \Delta x, j \Delta t)$, $f(x, t)=u(i, j)$ and applying forward difference quotients for both derivatives in (41), it is easy to verify that (41) can be expressed in the following form:

$$
\begin{aligned}
{\left[\begin{array}{l}
r(i, j) \\
s(i, j)
\end{array}\right]=} & {\left[\begin{array}{cc}
\left(1+a_{1} \Delta x\right) & \left(a_{1} a_{2}+a_{0}\right) \Delta x \\
0 & 0
\end{array}\right]\left[\begin{array}{l}
r(i-1, j) \\
s(i-1, j)
\end{array}\right] } \\
& +\left[\begin{array}{cc}
0 & 0 \\
\Delta t & \left(1+a_{2} \Delta t\right)
\end{array}\right]\left[\begin{array}{l}
r(i, j-1) \\
s(i, j-1)
\end{array}\right] \\
& +\left[\begin{array}{c}
b \Delta x \\
0
\end{array}\right] u(i-1, j)+\left[\begin{array}{l}
0 \\
0
\end{array}\right] u(i, j-1)
\end{aligned}
$$

with the initial conditions:

$$
s(i, 0)=p(i \Delta x), \quad r(0, j)=z(j \Delta t) .
$$

Now, taking

$$
\mathbf{x}(i, j)=\left[\begin{array}{l}
r(i, j) \\
s(i, j)
\end{array}\right],
$$

equation (43) can be expressed in the FMSLSS setting:

$$
\begin{aligned}
\mathbf{x}(i+1, j+1)= & {\left[\begin{array}{cc}
0 & 0 \\
\Delta t & \left(1+a_{2} \Delta t\right)
\end{array}\right] \mathbf{x}(i+1, j) } \\
& +\left[\begin{array}{cc}
\left(1+a_{1} \Delta x\right) & \left(a_{1} a_{2}+a_{0}\right) \Delta x \\
0 & 0
\end{array}\right] \mathbf{x}(i, j+1) \\
& +\left[\begin{array}{l}
0 \\
0
\end{array}\right] \mathbf{u}(i+1, j)+\left[\begin{array}{c}
b \Delta x \\
0
\end{array}\right] \mathbf{u}(i, j+1)
\end{aligned}
$$

with the initial conditions:

$$
\mathbf{x}(i, 0)=\left[\begin{array}{c}
-a_{2} p(i \Delta x) \\
p(i \Delta x)
\end{array}\right], \quad \mathbf{x}(0, j)=\left[\begin{array}{l}
z(j \Delta t) \\
q(j \Delta t)
\end{array}\right] .
$$

In view of (40) and (45), (38b) can be written as

$$
\mathbf{y}(i, j)=\mathbf{C x}(i, j),
$$

where

$$
\mathbf{C}=\left[\begin{array}{ll}
c_{1} & c_{2}
\end{array}\right]
$$

Now, consider the problem of anti-windup controller for the system represented by (46)-(49) with

$$
\begin{gathered}
a_{0}=0.2, \quad a_{1}=-1.8, \quad a_{2}=-0.5, \\
b=-0.194, \quad \Delta x=0.5, \quad \Delta t=1, \\
c_{1}=1, \quad c_{2}=0 .
\end{gathered}
$$


Consequently, the plant under consideration is described by (1) where

$$
\begin{gathered}
\mathbf{A}_{1}=\left[\begin{array}{cc}
0 & 0 \\
1 & 0.5
\end{array}\right], \quad \mathbf{A}_{2}=\left[\begin{array}{cc}
0.1 & 0.55 \\
0 & 0
\end{array}\right], \\
\mathbf{B}_{1}=\left[\begin{array}{l}
0 \\
0
\end{array}\right], \quad \mathbf{B}_{2}=\left[\begin{array}{c}
-0.097 \\
0.01
\end{array}\right], \\
\mathbf{C}=\left[\begin{array}{ll}
1 & 0
\end{array}\right] .
\end{gathered}
$$

For the above plant, the parameters of the stabilizing dynamic compensator (2) is given by

$$
\begin{aligned}
\mathbf{A}_{c 1} & =[0.2], & \mathbf{A}_{c 2} & =[0.3], \\
\mathbf{B}_{c 1} & =[0.01], & \mathbf{B}_{c 2} & =[0.01], \\
\mathbf{D}_{c} & =[11.12], & \mathbf{C}_{c} & =[0.1] .
\end{aligned}
$$

Assume that, the control signal injected to the plant is a saturated one characterized by (5) where

$$
-10 \leq u_{(l)}(i, j) \leq 10
$$

The resulting closed loop system is obtained by substituting (51) and (52) in (10a)-(10b). It is understood that the initial conditions of the closed loop system belong to (11). Using MATLAB LMI toolbox [36] and choosing $\alpha=0.5$, it is verified that (16)-(18) are feasible for the present example and the values of unknown parameters are obtained as

$$
\begin{gathered}
\mathbf{W}=\left[\begin{array}{ccc}
0.0810 & -0.0246 & -6.4152 \\
-0.0246 & 0.3181 & 14.7750 \\
-6.4152 & 14.7750 & 9850.1
\end{array}\right], \\
\mathbf{S}=\left[\begin{array}{cc}
2103800 & 0 \\
0 & 10.2344
\end{array}\right] \\
\mathbf{Z}=\left[\begin{array}{ll}
-114.7141 & -110.7475
\end{array}\right]
\end{gathered}
$$

In this example, the gain matrix

$$
\mathbf{E}_{c}=\mathrm{ZS}^{-1}=\left[\begin{array}{ll}
-0.0001 & -10.8212
\end{array}\right]
$$

Therefore, Theorem 1 assures that the system under consideration is asymptotically stable in the region given by the ellipsoid $\mathcal{\varepsilon}(\mathbf{P})=\left\{\boldsymbol{\xi} \in \mathfrak{R}^{n+n_{c}} ; \boldsymbol{\xi}^{T} \mathbf{P} \boldsymbol{\xi} \leq 2\right\}$.

\section{Conclusions}

A Lyapunov-based approach to design an antiwindup gain of 2D discrete systems with saturating controls in the FMSLSS setting is established. Stability conditions allowing the design of antiwindup loops, in both local and global contexts have been stated for this system. The proposed criterion is in LMI setting and can be efficiently solved using MATLAB LMI Toolbox $[35,36]$. Numerical examples are provided to illustrate the applicability of the presented results. Application of the proposed antiwindup controller design method is demonstrated through processes described by a Darboux equation [37-39].

The presented approach utilizes the 2D Lyapunov condition [23] which provides only sufficient condition for the stability and is not necessary. It is known that, unlike its 1D counterpart, the available Lyapunov-based approaches [23-27] provide only sufficient condition for the asymptotic stability of 2D linear discrete systems. The problem of determining necessary and sufficient conditions for the stability of $2 \mathrm{D}$ discrete saturated systems is very challenging. Further investigation is required to reduce or eliminate the gap between "sufficiency" and the "necessity" for a 2-D system to be stable, which occurs in the proposed approach.

\section{References}

[1] N. Kapoor, A. R. Teel, and P. Daoutidis, "An anti-windup design for linear systems with input saturation," Automatica, vol. 34, no. 5, pp. 559-574, 1998.

[2] Y. Y. Cao, Z. Lin, and D. G. Ward, "An antiwindup approach to enlarging domain of attraction for linear systems subject to actuator saturation," IEEE Transactions on Automatic Control, vol. 47, no. 1, pp. 140-145, 2002.

[3] G. Grimm, A. R. Teel, and L. Zaccarian, "The 12 antiwindup problem for discrete-time linear systems: definition and solutions," Systems and Control Letters, vol. 57, no. 4, pp. 356-364, 2008.

[4] P. C. Chen and J. S. Shamma, "Gain-scheduled $\ell$ 1-optimal control for boiler-turbine dynamics with actuator saturation," Journal of Process Control, vol. 14, no. 3, pp. 263-277, 2004.

[5] M. V. Kothare and M. Morari, "Multiplier theory for stability analysis of anti-windup control systems," Automatica, vol. 35, no. 5, pp. 917-928, 1999.

[6] T. Hu, A. R. Teel, and L. Zaccarian, "Stability and performance for saturated systems via quadratic and nonquadratic Lyapunov functions," IEEE Transactions on Automatic Control, vol. 51, no. 11, pp. 1770-1786, 2006.

[7] J. M. Gomes Da Silva Jr. and S. Tarbouriech, "Local stabilization of discrete-time linear systems with saturating controls: an LMI-based approach," IEEE Transactions on Automatic Control, vol. 46, no. 1, pp. 119-125, 2001.

[8] J. S. Shamma, "Anti-windup via constrained regulation with observers," in Proceedings of the American Control Conference (ACC '99), pp. 2481-2485, San Diego, Calif, USA, June 1999.

[9] J. M. Gomes Da Silva Jr. and S. Tarbouriech, "Anti-windup design with guaranteed regions of stability for discrete-time linear systems," Systems and Control Letters, vol. 55, no. 3, pp. 184-192, 2006.

[10] J. M. Gomes da Silva Jr. and S. Tarbouriech, "Antiwindup design with guaranteed regions of stability: an LMI-based approach," IEEE Transactions on Automatic Control, vol. 50, no. 1, pp. 106-111, 2005.

[11] L. L. Giovanini, "Model predictive control with amplitude and rate actuator saturation," ISA Transactions, vol. 42, no. 2, pp. 227-240, 2003.

[12] M. Zhang and C. Jiang, "Problem and its solution for actuator saturation of integrating process with dead time," ISA Transactions, vol. 47, no. 1, pp. 80-84, 2008.

[13] C. Roos, J. M. Biannic, S. Tarbouriech, C. Prieur, and M. Jeanneau, "On-ground aircraft control design using a 
parameter-varying anti-windup approach," Aerospace Science and Technology, vol. 14, no. 7, pp. 459-471, 2010.

[14] L. Zaccarian, Y. Li, E. Weyer, M. Cantoni, and A. R. Teel, "Antiwindup for marginally stable plants and its application to open water channel control systems," Control Engineering Practice, vol. 15, no. 2, pp. 261-272, 2007.

[15] T. S. Kwon and S. K. Sul, "Novel antiwindup of a current regulator of a surface-mounted permanent-magnet motor for flux-weakening control," IEEE Transactions on Industry Applications, vol. 42, no. 5, pp. 1293-1300, 2006.

[16] H. A. Fertik and C. W. Ross, "Direct digital control algorithm with anti-windup feature," ISA Transactions, vol. 6, pp. 317328, 1967.

[17] K. S. Walgama and J. Sternby, "Conditioning technique for multiinput multioutput processes with input saturation," IEE Proceedings D, vol. 140, no. 4, pp. 231-241, 1993.

[18] S. Tarbouriech and M. Turner, "Anti-windup design: an overview of some recent advances and open problems," IET Control Theory and Applications, vol. 3, no. 1, pp. 1-19, 2009.

[19] E. Fornasini and G. Marchesini, "Doubly-indexed dynamical systems: state-space models and structural properties," Mathematical Systems Theory, vol. 12, no. 1, pp. 59-72, 1978.

[20] T. Kaczorek, Two-Dimensional Linear Systems, Springer, Berlin, Germany, 1985.

[21] R. N. Bracewell, Two-Dimensional Imaging, Prentice-Hall Signal Processing Series, Prentice Hall, Englewood Cliffs, NJ, USA, 1995.

[22] N. K. Bose, Applied Multidimensional System Theory, Van Nostrand Reinhold, New York, NY, USA, 1982.

[23] T. Hinamoto, "2-D Lyapunov equation and filter design based on the Fornasini-Marchesini second model," IEEE Transactions on Circuits and Systems I, vol. 40, no. 2, pp. 102-109, 1993.

[24] T. Hinamoto, "Stability of 2-D discrete systems described by the fornasini-marchesini second model," IEEE Transactions on Circuits and Systems I, vol. 44, no. 3, pp. 254-257, 1997.

[25] W. S. Lu, "On a Lyapunov approach to stability analysis of 2-D digital filters," IEEE Transactions on Circuits and Systems I, vol. 41, no. 10, pp. 665-669, 1994.

[26] T. Ooba, "On stability analysis of 2-D systems based on 2-D Lyapunov matrix inequalities," IEEE Transactions on Circuits and Systems I, vol. 47, no. 8, pp. 1263-1265, 2000.

[27] D. Liu, "Lyapunov stability of two-dimensional digital filters with overflow nonlinearities," IEEE Transactions on Circuits and Systems I, vol. 45, no. 5, pp. 574-577, 1998.

[28] H. Kar and V. Singh, "An improved criterion for the asymptotic stability of 2-D digital filters described by the fornasinimarchesini second model using saturation arithmetic," IEEE Transactions on Circuits and Systems I, vol. 46, no. 11, pp. 1412-1413, 1999.

[29] H. Kar and V. Singh, "Stability analysis of 2-D digital filters described by the Fornasini-Marchesini second model using overflow nonlinearities," IEEE Transactions on Circuits and Systems I, vol. 48, no. 5, pp. 612-617, 2001.

[30] H. Kar and V. Singh, "Stability analysis of 1-D and 2-D fixed-point state-space digital filters using any combination of overflow and quantization nonlinearities," IEEE Transactions on Signal Processing, vol. 49, no. 5, pp. 1097-1105, 2001.

[31] V. Singh, "Stability analysis of 2-D discrete systems described by the Fornasini-Marchesini second model with state saturation," IEEE Transactions on Circuits and Systems II, vol. 55, no. 8, pp. 793-796, 2008.
[32] H. Kar and V. Singh, "Robust stability of 2-D discrete systems described by the Fornasini-Marchesini second model employing quantization/overflow nonlinearities," IEEE Transactions on Circuits and Systems II, vol. 51, no. 11, pp. 598-602, 2004.

[33] V. Singh, "Robust stability of 2-D digital filters employing saturation," IEEE Signal Processing Letters, vol. 12, no. 2, pp. 142-145, 2005.

[34] A. Hmamed, F. Mesquine, F. Tadeo, M. Benhayoun, and A. Benzaouia, "Stabilization of 2D saturated systems by state feedback control," Multidimensional Systems and Signal Processing, vol. 21, no. 3, pp. 277-292, 2010.

[35] S. Boyd, L. EI-Ghaousi, E. Feron, and V. Balakrishnan, Linear Matrix Inequalities in Systems and Control Theory, SIAM, Philadelphia, Pa, USA, 1994.

[36] P. Gahinet, A. Nemirovski, A. J. Laub, and M. Chilali, LMI Control Toolbox-For Use with MATLAB, MathWorks, Inc., Natic, Mass, USA, 1995.

[37] W. Marszalek, "Two-dimensional state-space discrete models for hyperbolic partial differential equations," Applied Mathematical Modelling, vol. 8, no. 1, pp. 11-14, 1984.

[38] C. Du, L. Xie, and C. Zhang, "Hळ control and robust stabilization of two-dimensional systems in Roesser models," Automatica, vol. 37, no. 2, pp. 205-211, 2001.

[39] J. S. H. Tsai, J. S. Li, and L. S. Shieh, "Discretized quadratic optimal control for continuous-time two-dimensional systems," IEEE Transactions on Circuits and Systems I, vol. 49, no. 1, pp. 116-125, 2002. 


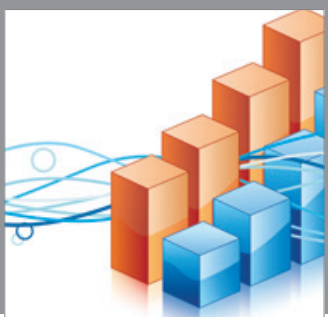

Advances in

Operations Research

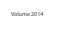

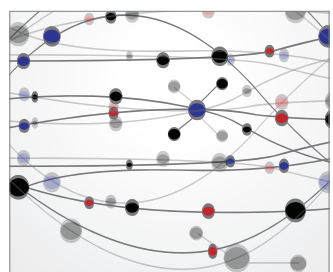

\section{The Scientific} World Journal
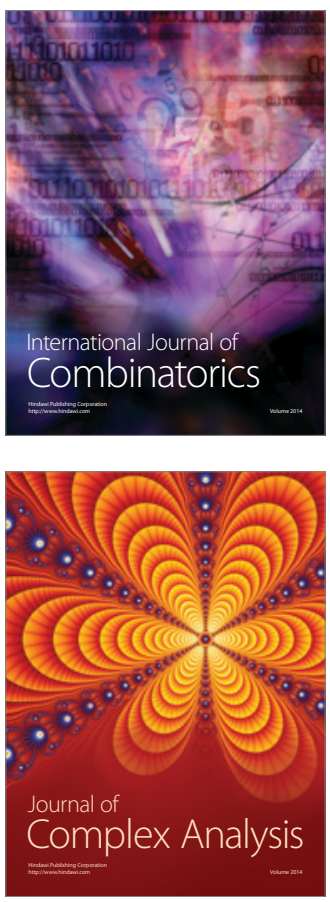

International Journal of

Mathematics and

Mathematical

Sciences
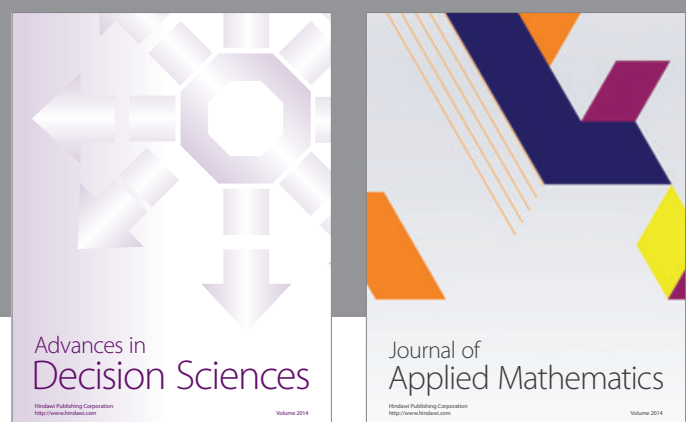

Journal of

Applied Mathematics
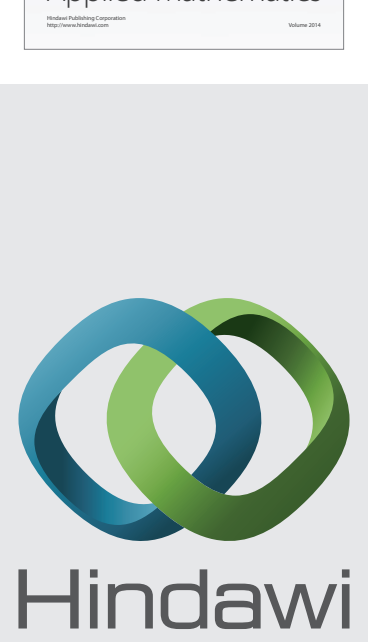

Submit your manuscripts at http://www.hindawi.com
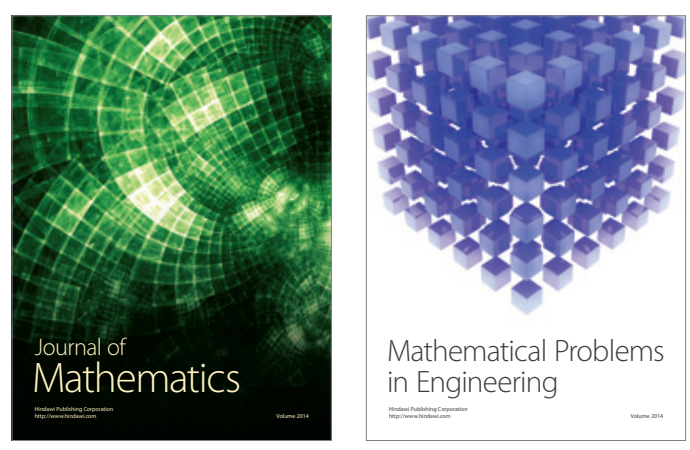

Mathematical Problems in Engineering
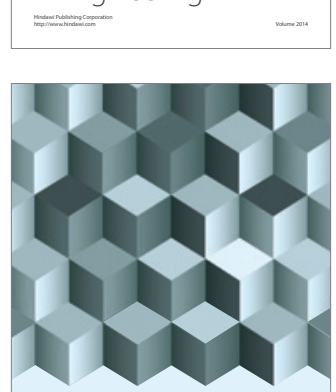

Journal of

Function Spaces
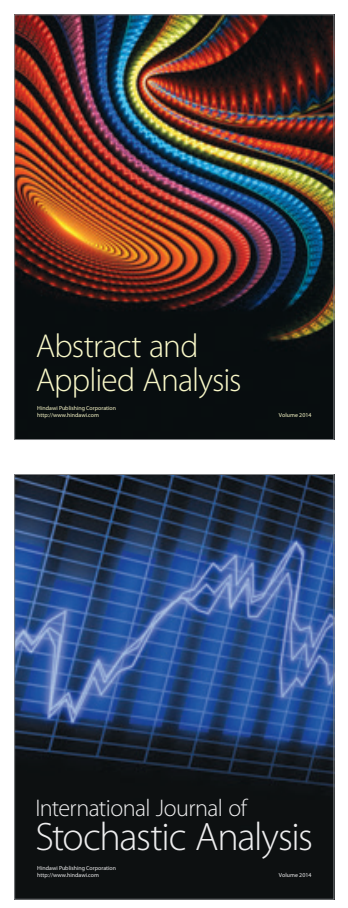

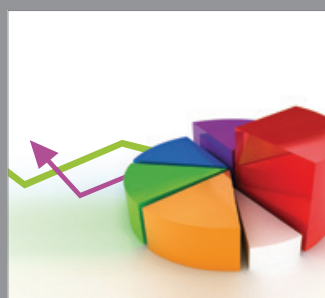

ournal of

Probability and Statistics

Promensencen
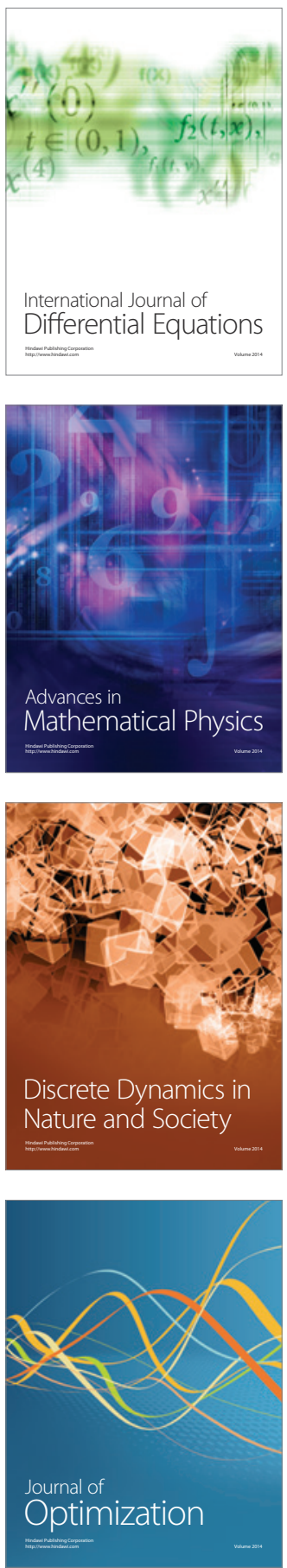